\title{
Reconstruction of a three-dimensional bubble surface from high-speed orthogonal imaging of dilute bubbly flow
}

\author{
M. Honkanen \\ Tampere University of Technology, \\ Energy and Process Engineering, Finland
}

\begin{abstract}
This paper presents image analysis algorithms to reconstruct a three-dimensional gas bubble surface from the time series of two orthogonal projections and to also recognize bubble outlines when the bubbles are partly overlapping in the image. Overlapping bubble images raise a large problem for three-dimensional, tomographic imaging of bubbly flows. Here, a novel approach to recognize the outlines of irregularly-shaped, overlapping bubbles based on the overlapping object recognition algorithm is presented.

The main objective is to investigate the accuracy of a back-light imaging technique to measure bubble properties from a) a single projection or b) from two, orthogonal projections. The time series data of the bubble surface area, volume and orientation, location, velocity and trajectory direction is obtained. Temporal fluctuations in the measured bubble volume reveal the measurement accuracy in the case of a bubble swarm rising from a single orifice.
\end{abstract}

Keywords: image analysis, three-dimensional imaging, high-speed imaging, bubble sizing, overlapping object recognition, optical tomography.

\section{Introduction}

The direct imaging technique for particle and bubble tracking and sizing (e.g., [1, $2]$ ) is not yet widespread; it has only become feasible with high-resolution CCD cameras and fast image processing possibilities [3]. However, direct imaging potentially offers size estimates of irregularly-shaped objects. Difficulties arise with the definition of the size of the observation volume and with optical access, 
and a compromise is made between observation area and size resolution. Therefore, the direct imaging technique is more applicable for sizing larger particles in dilute systems [3].

In the experimental images of bubbly flows, image segments are often created by groups of bubbles and not by individual bubbles, i.e. objects. If a grey-scale thresholding method is applied and two or more objects overlap in the image, they are detected as one big object and only one size and velocity is measured for the whole group. This causes errors in the measured size and velocity distributions of the studied objects. Therefore, overlapping object recognition algorithms [4-10] are necessary to increase measurement accuracy and sample rate by recognizing individual, in-focus objects. Utilization of the specific pattern recognition algorithms is discussed in [10].

The properties of irregularly-shaped bubbles are difficult to measure. Image analysis retrieves the properties of one or several two-dimensional projections of the bubble. The relation between the three-dimensional properties (bubble volume, characteristic size, surface area, orientation) and the measured image properties (projected area diameter, perimeter, major and minor dimensions) must be determined. Bubble volume can be estimated as a volume of an equivalent sphere with a diameter equal to the projected area diameter of the bubble image. A more precise estimation is obtained assuming that the bubble has a symmetrical shape along its vertical axis, e.g. ellipsoidal shape, and that the third main axis of the ellipsoid is predicted with the axes visual in the image [4].

Typically, image-based bubble sizing techniques rely on single projection. Here, two orthogonal views of bubble swarm rising from a single orifice are obtained with a high-speed camera, two mirrors and a LED back-light. Two orthogonal views (direct and mirror images) are geometrically mapped to the same scale and a three-dimensional Lagrangian bubble tracking algorithm is utilized to obtain time series data of bubble surface area, volume and orientation, location, velocity and trajectory direction. Temporal fluctuations in measured bubble volume reveal the measurement accuracy. For comparison, bubble volume is measured from a single view based on common assumptions on bubble shape.

\section{Measurement setup}

High-speed digital imaging setup is used to visualize the rise of air bubbles in stagnant tap water, in a bubble column. The flow is illuminated with a continuous, white LED array. High-speed CMOS camera (Photron SA1) acquires megapixel images at the speed of 1000 frames per second. Two mirrors are placed at 45 degrees angles on both sides of the bubble column to provide also the side view of the column with the same camera. Photograph of the measurement setup without mirrors is shown in Fig. 1.

Camera image is geometrically calibrated with a measuring rod placed in the middle of the column. Field of view is chosen to cover the column cross-section in the front view and in the side view through the side mirror. Image scaling of $0.135 \mathrm{~mm}$ per image pixel is obtained for the front view and $0.149 \mathrm{~mm} /$ pixel for 
the side view. Figure 2 shows images of measuring rod and bubbles in the measurement volume. The aperture of the objective (Sigma 105mm macro) is closed to $\mathrm{f}^{\sharp} 16$ to view all bubbles in-focus in both views. The front and side views are cropped from the original image and the front-view image is downsampled to match the size of the side-view image. That provides us front-view and side-view images whose vertical coordinates are equal. The vertical location and dimension of each bubble image can be approximated equal in both views, because bubbles rise up in the middle of the column.

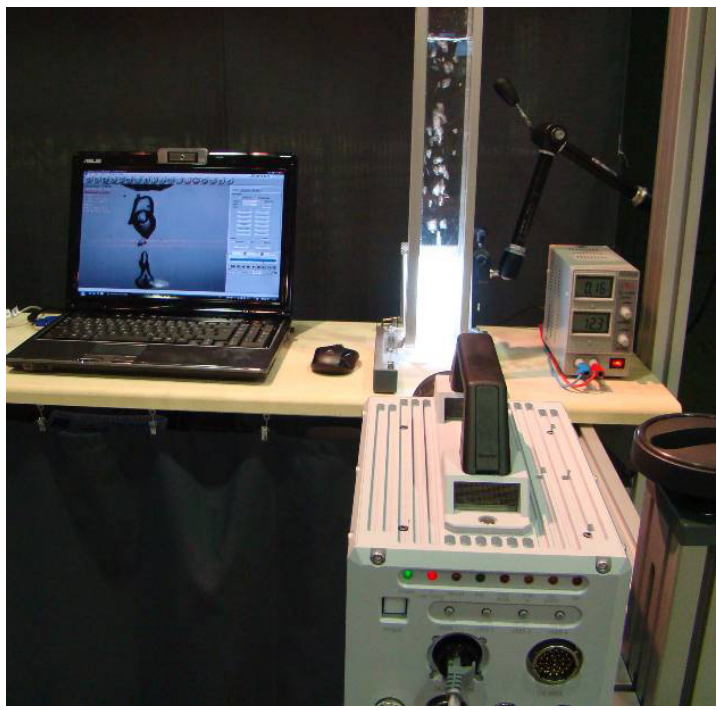

Figure 1: Measurement setup of bubble column, HS-camera and LED light.
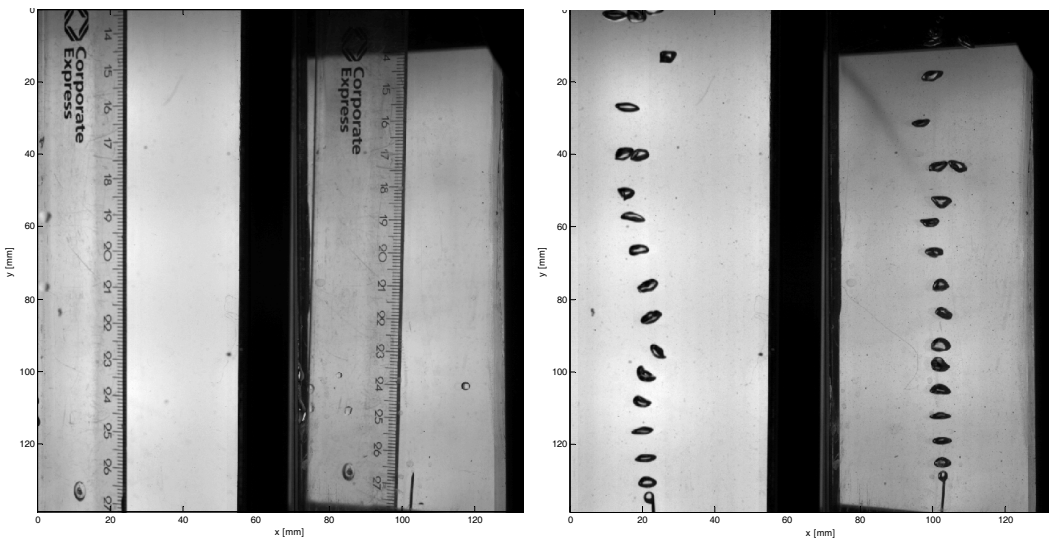

Figure 2: $\quad$ Image of measuring rod on the left and an experimental image on the right. 


\section{Image analysis}

High-speed image sequences are analyzed automatically with the following image analysis algorithms. Algorithms can be divided into 4 groups: image segmentation, overlapping object recognition, three-dimensional Lagrangian particle tracking, reconstruction of three-dimensional bubble surface and computation of bubble properties. Image processing and analysis algorithms are implemented in MATLAB computing software.

\subsection{Image segmentation}

Firstly, bubble shadows have to be distinguished from image background. An instantaneous image background image $I_{b g}$ is computed individually for each image utilizing a sliding (40x40 pixel) greyscale maximum filter. The background greyscale level is estimated as $90 \%$ of the sliding maximum value. Niblack [11] has presented an image segmentation method that scales the greyscale threshold value with a local $(15 \times 15$ pixel $)$ standard deviation of the image greyscales $I_{\text {std }}$.

$$
I_{t h r}=I_{b g}-0.12 \cdot I_{s t d},
$$

The image pixels with greyscale value lower than the value in the greyscale threshold image $I_{t h r}$, belong to bubble segments. Bubble segments are filled with morphological filter to include also the bright holes of bubble shadows in the bubble segments. Bubble segments are labelled and their properties are computed individually from both views. Bubble image properties include centre point coordinates, bounding box dimensions, projected area, perimeter, major and minor axis, aspect ratio and orientation. The major and minor axes (i.e. principal components) of the bubble image are obtained with direct least square ellipse fitting method [12].

\subsection{Analysis of overlapping bubble images}

Bubbles may overlap in one or both of the projections as can be seen in the example image in Fig. 2. The cases of bubble image overlap are recognized via solidity criterion: A bubble segment is bounded with a convex line by minimizing the length of the line. If the solidity (i.e. the ratio of the segment area and the area inside the convex line) is less than the solidity-threshold value ( 0.9), the segment must include more than one bubble.

Image segments of overlapping bubbles are analyzed with a watershed algorithm [8] or with a contour curvature-based OOR algorithm [9] that fits ellipses on contour arcs or produces breaklines between the connecting points of overlapping bubbles. The accuracy of contour curvature computation is further improved utilizing the adaptive curvature functions presented by Urdiales et al. [13]. Figure 3 shows the overlapping object recognition results of the three algorithms for the example image (Fig. 2). Watershed segmentation is sensitive to the changes on the bubble outline resulting in recognition of too many bubbles in case of the side view in Fig. 3a. The contour-curvature based OOR algorithms provide correct results. The advantage of breakline detection method in 
comparison to ellipse fitting method is clear in this case of non-ellipsoidal bubble images. However, watershed and breakline detection methods cut off overlapping segment parts, whereas the ellipse fitting method can estimate the full sizes of partly overlapping bubbles.

a)

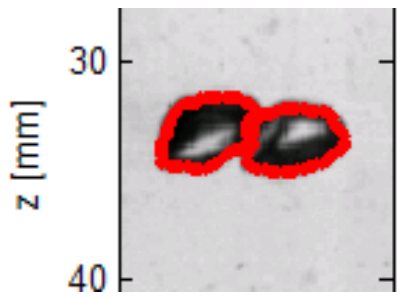

b)

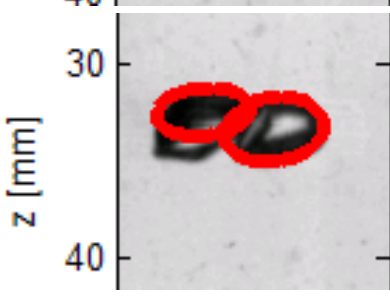

c)

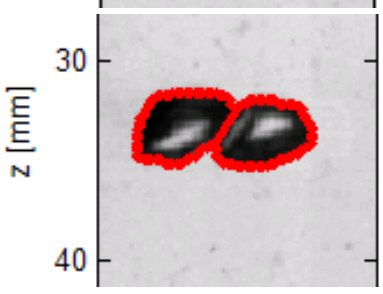

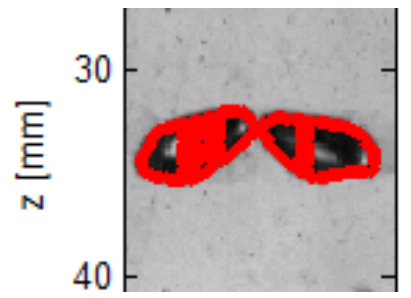

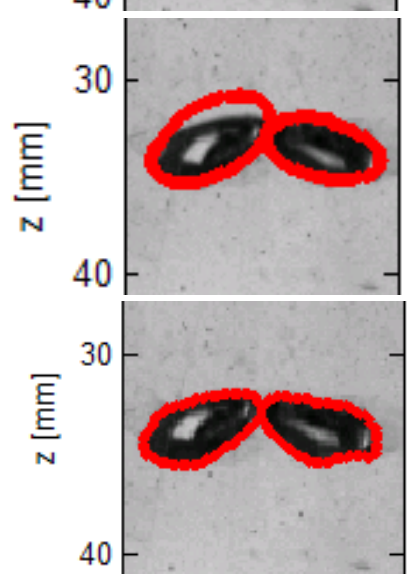

Figure 3: Analyzed segment of overlapping bubbles by a) Watershed algorithm or by Contour-curvature analysis with b) ellipse fitting and c) with breakline detection.

\subsection{Three-dimensional Lagrangian particle tracking}

There are several ways to implement three-dimensional Lagrangian particle tracking for a high-speed image sequence [14]. Guezennez et al. [15] proposed to carry out particle tracking in time prior to the projective matching especially in case of orthogonal imaging. They concluded that Lagrangian particle tracking in two-dimensions followed with the matching of particle trajectories between two projections is easier than individual particle matching followed by threedimensional Lagrangian particle tracking. In this study, bubble concentration is so low that bubble matching and tracking tasks are trivial. Both tasks are realized as least squares error minimization functions. Projective matching of recognized bubble images relies on the similarity of the vertical location and dimension of each bubble image in both views. Note that in the case of perfect overlap of bubbles in one of the views, the projective matching can return several matches for single bubble projection. Three-dimensional Lagrangian particle tracking 
algorithm assumes constant rise velocity and size of each bubble allowing maximum of $20 \%$ variation in the computed pseudo-distance $s$ :

$$
s_{k, j}=\sqrt{\left(x_{i, e}(k)-x_{i}(j)\right)^{2}+\left(y_{i, e}(k)-y_{i}(j)\right)^{2}+\left(z_{i, e}(k)-z_{i}(j)\right)^{2}+\left(d_{i-1}(k)-d_{i}(j)\right)^{2}}
$$

where bubble trajectory $k$ is compared to the bubble $j$ in image $i$. The bubble coordinate estimates $\left(x_{i, e}, y_{i, e}, z_{i, e}\right)$ for image $i$ are obtained from bubble trajectory assuming constant rise velocity of the bubble. $d_{i-1}$ is the previously measured diameter of bubble $k$. All possible bubble trajectories are compared to the bubble values $\left(x_{i}, y_{i}, z_{i}\right.$ and $\left.\mathrm{d}_{\mathrm{i}}\right)$ in the next image frame and the match is found minimizing the pseudo-distance, eqn. (2). Bubble properties along the bubble trajectory are stored only in frames, in which the algorithm has returned a consistent match. Bubble tracking is not stopped if the correct match is not found in the next image frame, but in that case the search is continued to the subsequent frames and the particle location estimate is updated.

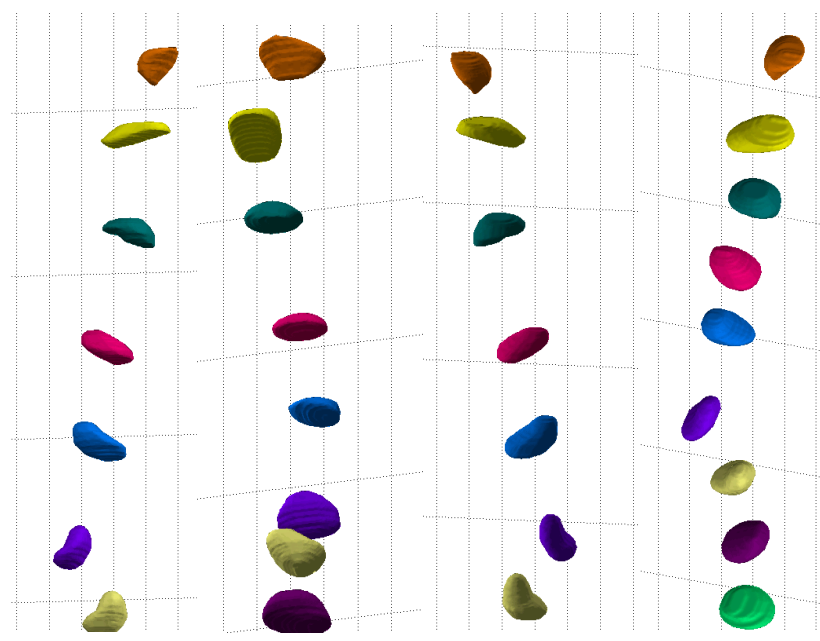

Figure 4: Four views of an analyzed bubble image. Bubbles are colourcoded so as to recognize the same bubbles in four views.

\subsection{Reconstruction of a three-dimensional bubble surface}

Following steps are taken to reconstruct a three-dimensional bubble surface from two projections at each time step:

a) A three-dimensional bounding box is generated for each bubble assuming that the scaled, vertical dimensions equal in both projections.

b) The bounding box is scanned with a horizontal plane that is moved along the vertical axis from top to bottom. Three-dimensional bubble image is reconstructed based on ellipses fitted on the horizontal planes, where the ellipse location and main axes correspond to the bubble image centre locations and widths in both projections, at the height of the horizontal plane. The ellipse orientation remains unknown, because the horizontal plane is not visible in neither of the two projections. 
c) The $3 \mathrm{~d}$ bubble image is smoothed with a $3 \times 3 \times 3$ smoothing filter to provide continuous bubble surface.

d) The $3 \mathrm{~d}$ bounding box is placed in the coordinate system of the measurement volume and an iso-surface of the $3 \mathrm{~d}$ bubble image is formed in that location. The colour of the surface is defined individually for each bubble trajectory, so every bubble is colour-coded making it easier to follow it in image sequence. Finally, lightings are added to the $3 \mathrm{~d}$ image.

Figure 4 shows four views of a single $3 \mathrm{~d}$ bubble image in different angles. Horizontal planes are clearly visible on the reconstructed bubble surface because of the low spatial resolution of the measurement. However, a larger field of view allows inspection of bubbles over $13 \mathrm{~cm}$ vertical distance.

\subsection{Computation of bubble properties}

The bubble properties are measured both from single projections and from two, orthogonal projections. Volume-equivalent diameter is chosen to represent the characteristic size of a bubble. It can be computed from a single projection a) as a projected area diameter $d_{\text {projArea }}$ of the bubble image, eqn. (3), or b) assuming an ellipsoidal shape or more precisely oblate spheroid, setting the second horizontal axis of the ellipsoid equal to the visible horizontal axis [4], eqn. (4).

$$
\begin{gathered}
d_{\text {projArea }}=2 \cdot \sqrt{\frac{A_{\text {proj }}}{\pi}} \text { (3) } \quad d_{\text {projPCA }}=\left(a^{2} \cdot b\right)^{1 / 3} \\
d_{\text {est }}=(a \cdot b \cdot c)^{1 / 3} \\
\text { (5) } d_{\text {num }}=\left(\frac{6 \cdot V}{\pi}\right)^{1 / 3}
\end{gathered}
$$

Computation of volume-equivalent diameter from two orthogonal projections can rely on ellipsoidal shape-assumption estimating the diameter with the main axes of the ellipsoid by eqn. (5), or it can be computed directly from the $3 \mathrm{~d}$ bubble image counting the bubble volume $V$ in voxel by voxel-basis and following eqn. (6).

Measurement of bubble surface area is more uncertain than the volume, because it can vary in time and the bubble surface is curved in all dimensions. Bubble surface area can be estimated from surface areas of volume-equivalent sphere and ellipsoid, and it can be computed directly from the reconstructed $3 \mathrm{~d}$ bubble image. Bubble orientation is provided in two projections, but not in the horizontal (x-y) plane. For that, a third view would be necessary.

\section{Results and discussion}

Three-dimensional, time-resolved measurement results are difficult to present in a two-dimensional, stationary form. An attempt is made in Figure 5 to present the three-dimensional motion of bubbles. Colour-coded bubble trajectories are shown in front and side views on left and the $3 \mathrm{~d}$ bubble image with the same colour-coding is shown on right, where column's front-right corner is in front. The 4-mm bubbles rise up at about $0.3 \mathrm{~m} / \mathrm{s}$. Every bubble is sampled about 400 times during its $13 \mathrm{~cm}$ rise from the orifice. 

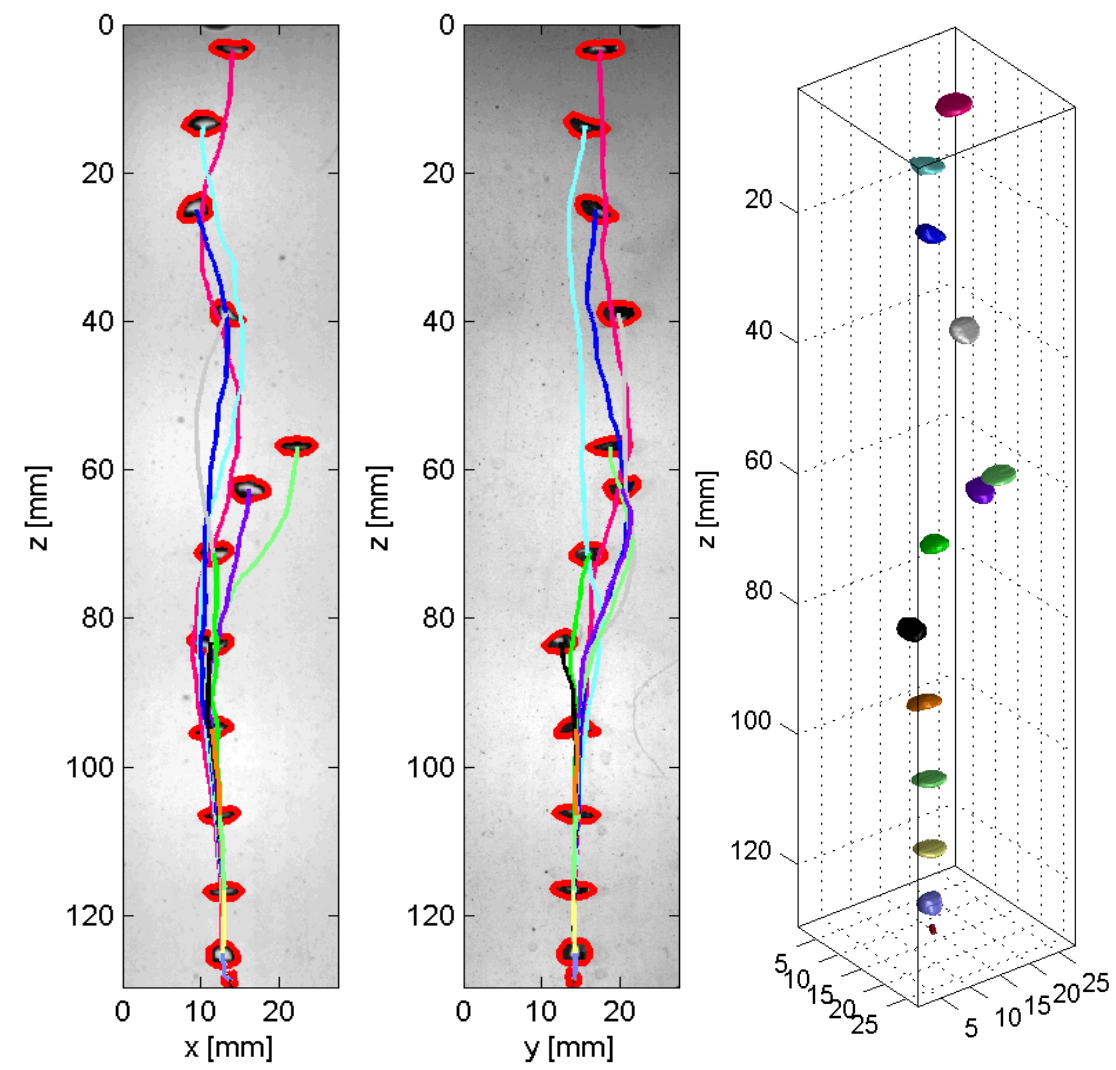

Figure 5: Two views of bubbles with measured outlines and colour-coded trajectories and the reconstructed $3 \mathrm{~d}$ image of bubbles.

Time series measurement results enable statistical analysis of measurement accuracy. The volume of an air bubble can be assumed constant during the $13 \mathrm{~cm}$ bubble rise in water. The volume-equivalent bubble diameter is measured in six different ways. Time series results of bubble velocity, surface area and volumeequivalent diameter for two example bubbles are shown in Figure 6.

Average and standard deviation values of six measures of volume-equivalent bubble diameter are presented in Table 1 for 25 bubble trajectories. Measures from single projection have standard deviation of about $7 \%$ and the measure based on ellipsoidal-shape assumption performs slightly better than a projected area diameter. The same confidence interval is found by Rodriguez-Rodriguez et al. [15], who investigated errors produced by processing a two-dimensional image of the three-dimensional ellipsoidal bubble. They concluded that typically the sizing error is bounded by $0.85<\mathrm{d}_{\tau} / \mathrm{d}_{\mathrm{p}}<1.08$. In our case, the projected area diameter clearly underestimates the bubble size, whereas ellipsoid-assumption overestimates it. The measures from two projections are clearly more accurate than the estimates from single projections. Direct $3 \mathrm{~d}$ bubble volume computation 

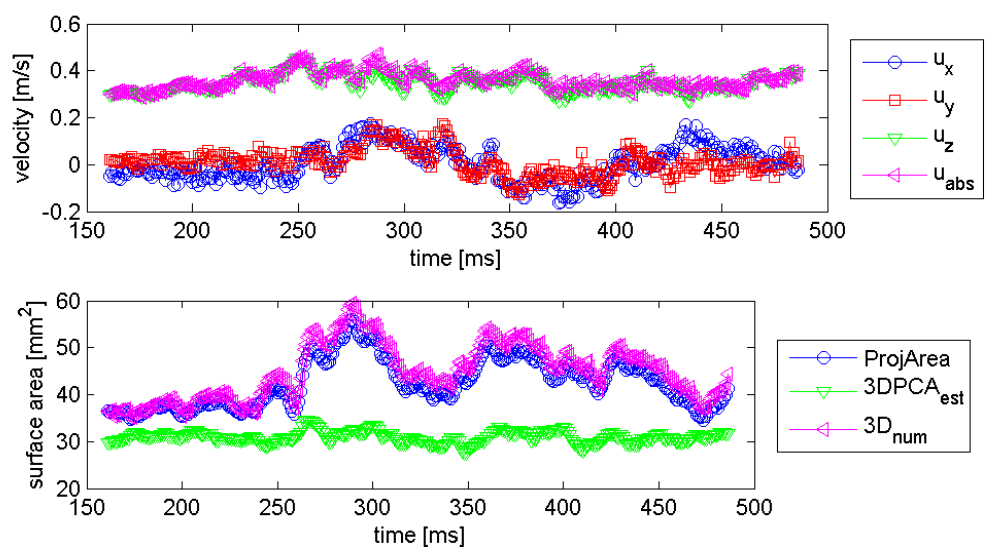

a)
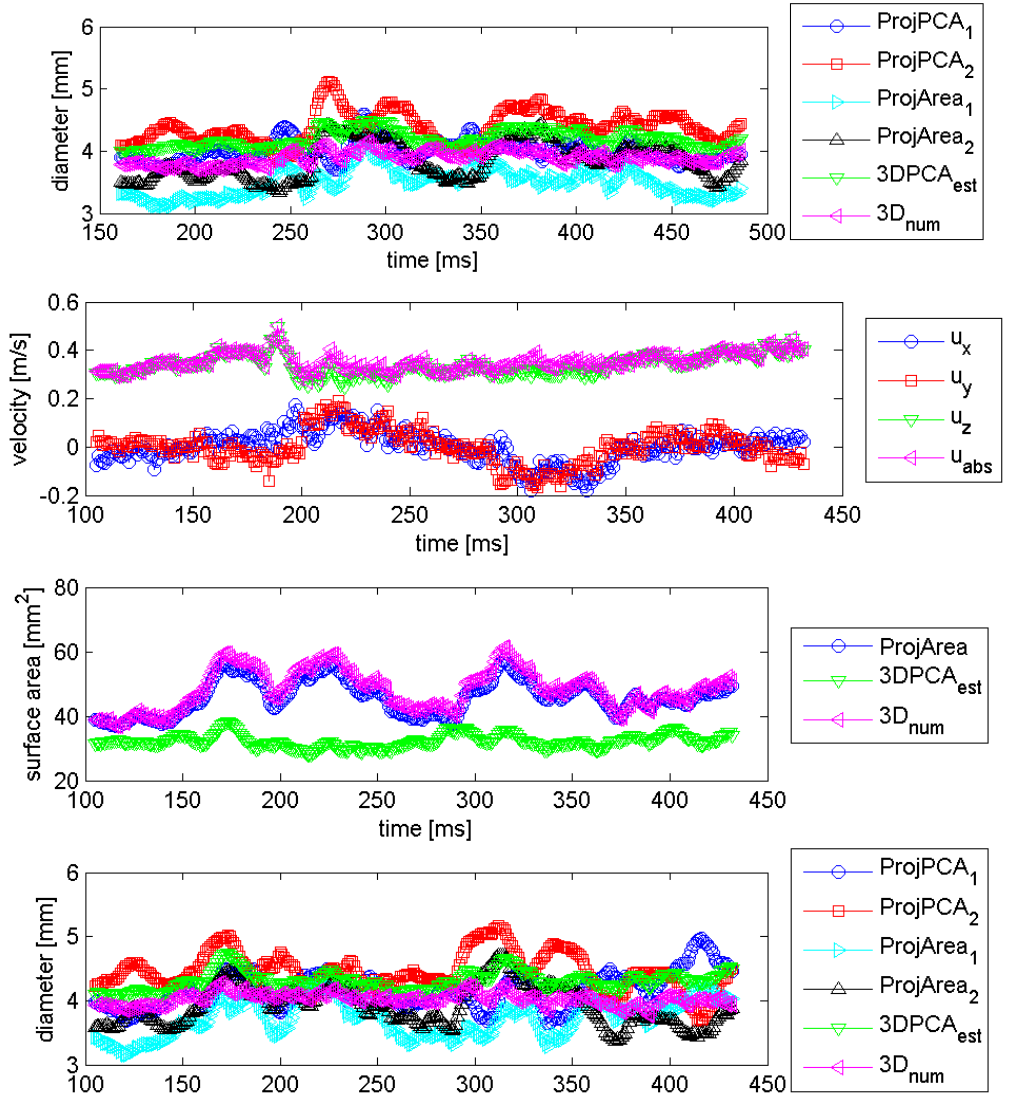

b)

time [ms]

Figure 6: Time series of bubble velocity, surface area and volume-equivalent diameter for example bubbles (a) and (b). 
provides standard deviation of $0.1 \mathrm{~mm}$, which is only $2 / 3$ image pixels. The principal axes of $3 d$ ellipsoid return a size measure with STD of 0.168 pixels, which is a good result. This shows that direct least squares ellipse fit returns reasonable size estimates also for irregularly-shaped bubbles with an ellipse-like shape.

Surface area of a bubble is measured a) as the area of the fitted ellipsoid, $3 D$ $P C A_{\text {est }}$, b) as the area of the sphere with a diameter equal to the mean of two projected area diameters, ProjArea, and c) directly from the $3 \mathrm{~d}$ bubble image. The $3 \mathrm{~d}$ bubble image is reconstructed from two projections and therefore, the projected areas relate to the $3 \mathrm{~d}$ bubble image providing nearly the same surface area. As expected, the surface area of a fitted ellipsoid is clearly smaller than the true surface area of a bubble.

Table 1: $\quad$ Statistics of measured volume-equivalent bubble diameter.

\begin{tabular}{|l|c|c|}
\hline Size measure & Average [mm] & Standard deviation [mm] \\
\hline Measures from two projections: & & \\
\hline Computed from 3d image, $\mathrm{d}_{\text {num }}$ & 3.959 & 0.104 \\
\hline 3d ellipsoid-assumption, $\mathrm{d}_{\text {est }}$ & 4.259 & 0.168 \\
\hline Measures from single projection: & & 0.304 \\
\hline Projected area diameter $\mathrm{d}_{\text {projArea 1 }}$ & 3.683 & 0.273 \\
\hline Projected area diameter $\mathrm{d}_{\text {projArea 2 }}$ & 3.882 & 0.289 \\
\hline Projected principal axes $\mathrm{d}_{\text {projPCA 1 }}$ & 4.127 & 0.281 \\
\hline Projected principal axes $\mathrm{d}_{\text {projPCA 2 }}$ & 4.402 & \\
\hline
\end{tabular}

Bubble formation and detachment from the orifice is interesting. Figure 7 shows time series data of a bubble during the first $100 \mathrm{~ms}$ of its trajectory. The bubble is first recognized when its size is $2 \mathrm{~mm}$ in diameter and it is still attached to the orifice. Its size increases linearly $35 \mathrm{~ms}$ until it detaches from the orifice and starts rising up to the water surface. The detachment causes a peak in the rise velocity. The surface area of a bubble has its maximum value at the time of the detachment. After that the surface area decreases and starts oscillating as seen in Fig. 6. All volume-equalized bubble diameter measures, except directly computed value $\left(3 D_{\text {nиm }}\right)$, overestimate bubble size, when the bubble is attached to the orifice.

\section{Conclusions}

The presented high-speed orthogonal imaging approach is simple and effective method to measure gas bubble properties in three-dimensions. The threedimensional bubble surface is reconstructed from two projections and the 
bubbles are tracked $13 \mathrm{~cm}$ long path from the orifice. The volume-equivalent bubble diameter is measured with a sub-pixel accuracy having relative error of only $2.5 \%$. Also two-dimensional image-based measurements provide accurate estimates of bubble size with a root mean square error of about $7 \%$. However, third view would be necessary to measure the $3 \mathrm{~d}$ orientation of a bubble. The presented overlapping object recognition algorithms enable the analysis of threedimensional bubble trajectories also in more complex bubbly flows. Future work is to apply the presented imaging techniques in different applications.
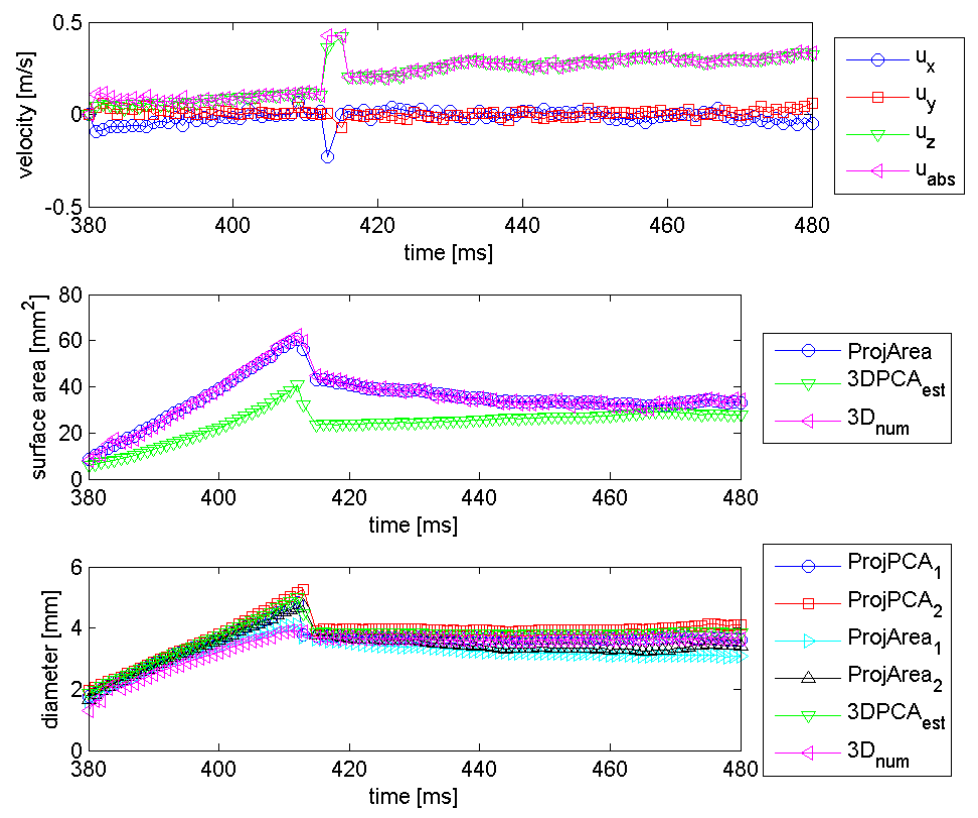

Figure 7: Time series of bubble velocity, surface area and volume-equivalent diameter during the bubble generation and ejection from the orifice.

\section{Acknowledgement}

The financial support of Academy of Finland is gratefully acknowledged.

\section{References}

[1] Nishino, K., Kato, H., Torii, K. Stereo Imaging for simultaneous measurement of size and velocity of particles in dispersed two-phase flow. Meas Sci Technol 11, pp.633-645, 2000.

[2] Pan, X. H., Luo, R., Yang, X. Y., Yang, H. J. Three-dimensional particle image tracking for dilute particle-liquid flows in a pipe. Meas Sci Technol 13, pp. 1206-1216, 2002. 
[3] Damaschke, N., Nobach, H., Nonn, D. I., Semidetnov, N., Tropea, C. Multi-dimensional particle sizing techniques. Exp Fluids 39, pp. 336-350, 2005.

[4] Bröder, D., Sommerfeld, M. Planar shadow image velocimetry for the analysis of the hydrodynamics in bubbly flows. Meas Sci Technol, 18, pp. 2513-2528, 2007.

[5] Berg van den, E. H., Meesters, A. G., Kenter, C. A., Schlager, W. Automated separation of touching grains in digital images of thin sections. Computers \& Geosciences 28, pp. 179-190, 2002.

[6] Pla, F. Recognition of Partial Circular Shapes from Segmented Contours. Computer Vision and Image Understanding vol. 63, pp. 334-343, 1996.

[7] Shen, L., Song, X., Iguchi, M., Yamamoto, F. A method for recognizing particles in overlapped particle images. Pattern Recognition Letters 21, pp. 21-30, 2000.

[8] Meyer, Fernand, Topographic distance and watershed lines. Signal Processing, 38, pp. 113-125, 1994.

[9] Honkanen, M., Saarenrinne, P., Stoor, T. \& Niinimäki, J. Recognition of highly overlapping ellipse-like bubble images. Meas Sci Technol, 16, pp. 1760-1770, 2005.

[10] Honkanen, M. Direct optical measurement of fluid dynamics and dispersed phase morphology in multiphase flows. Univ. print, Tampere, 2006, http://dspace.cc.tut.fi/dpub/handle/123456789/78 .

[11] Niblack, W. An Introduction to Digital Image Processing, pp. 115-116, Prentice Hall, 1986.

[12] Fitzgibbon, A., Pilu, M., Fisher, R. B. Direct least square fitting of ellipses. Pattern Analysis and Machine Intelligence, IEEE Transactions 21, pp. 476 $-480,1999$.

[13] Urdiales, C., Bandera, A., Sandoval, F. Non-parametric planar shape representation based on adaptive curvature functions. Pattern Recognition 35, pp. 43-53, 2002.

[14] Ouellette, N.T., Xu, H. \& Bodenschatz, E. A quantitative study of threedimensional Lagrangian particle tracking algorithms. Exp Fluids, 40, pp. 301-313, 2006.

[15] Guezennec, Y. G., Brodley, R. S., Trigui, N., Kent, J. C., Algorithms for fully automated three-dimensional particle tracking velocimetry. Exp Fluids 17, pp. 209-219, 1994.

[16] Rodriguez-Rodriguez, J., Martinez-Bazan, C., Montanes, J. L. A novel particle tracking and break-up detection algorithm: application to the turbulent break-up of bubbles. Meas Sci Technol 14 1328-1340, 2003. 